Socially Thermoregulated Thinking: How Past Experiences Matter in Thinking about Our Loved Ones

\author{
Hans IJzerman \\ Université Grenoble Alpes \\ Lison Neyroud \\ Université Grenoble Alpes \\ Rémi Courset \\ Université Grenoble Alpes
}

Michel Schrama

Independent Researcher

Jorick Post

Tilburg University

Tila M. Pronk

Tilburg University

This manuscript is in press at Journal of Experimental Social Psychology. It may not reflect the final version - cite at own risk! 


\begin{abstract}
Body temperature regulation is of crucial importance for nonhuman and human animals. Because other animals are crucial in helping to regulate body temperature, temperature differences likely determine how humans think about their social environment. Since 2008, the psychological literature on social thermoregulation has flourished with approximately 80 reports, ranging from economic decision-making to self-regulation. However, questions have arisen to its robustness and about underlying mechanisms, particularly in relation to differences in past relationship experiences. In this report, the authors used an inductive approach, exploring individual differences to identify items that alter the temperature-social thought relationship in a pilot (Study 1), and confirming the effects in Study 2 (total $N$ for 1 and $2=366$ ), both of which were not pre-registered. After a first review with the present journal, we preregistered our replication and successfully replicated our effects in a French sample $(N=350)$. Coldness (vs. warmth) makes people think about closer others when past relationship experiences were positive, while the reverse is true for negative past relationship experiences. These robust results provide future directions for the field of social thermoregulation.
\end{abstract}




\section{Socially Thermoregulated Thinking: How Past Experiences Matter in Thinking about}

\section{Our Loved Ones}

The regulation of one's body temperature is crucial for one's survival. In fact, across animals, the co-regulation of temperature has been identified as a crucial factor in a species' continued existence (Ebensberger, 2001). In monkeys (McFarland et al., 2015) and humans (Inagaki et al., in press), higher core temperature relate to the size of the social network. While the effect of co-regulation of temperature has been studied in animals for decades (IJzerman et al., 2015a), research on social thermoregulation in humans only commenced in 2008. The importance for survival is clear, but do fluctuations in temperature affect how we think about significant others? And does this relationship rely on past experiences with close relationships?

Over the past few years, research on social thermoregulation in humans has mushroomed. With the first article in 2008, Williams and Bargh showed that people rate others as more sociable and behave more generously when holding something warm (vs. cold). Since then, 80 published and unpublished reports have appeared that relate social thermoregulation to diverse domains, such as prosocial behavior (Williams \& Bargh, 2008), self-regulation (Zhang \& Risen, 2014), social exclusion (IJzerman et al., 2012), relationships with consumer products (IJzerman et al., 2015b) and economic decision-making (Van Acker et al., 2016). Nevertheless, there are three important reasons to investigate this issue. First, a recent report suggests that the results may not be as robust as previously supposed (e.g., LeBel \& Campbell, 2013; Lynott et al., 2014; but see Schilder et al., 2014). The first goal of this article is thus to revisit whether temperature effects are robust. The second goal is to investigate whether temperature effects extend to the recall of close others. 
Third, an important factor to consider when interpreting these main effects of social thermoregulation is past experiences in (close) relationships. Results, so far, are inconsistent. IJzerman et al. (2013) found that securely (and not insecurely) attached children became more prosocial in a warmer (vs. colder) condition. Vess (2012) found that after thinking about a romantic breakup, the anxiously attached were more likely to desire warm foods (but see Lebel \& Campbell, 2013), yet, moderation by attachment was not found in another replication report (Schilder et al., 2015). To address this inconsistency, we explored whether the relationship between temperature and accessibility of close others is moderated by past experiences in close relationships. Specifically, we investigated whether warmth activates thoughts of close others and whether this differs for those who have positive (vs. negative) experiences in close relationships.

We used an inductive (i.e., data-driven) approach to generate subsequent predictions (Dotsch \& Todorov, 2012). More specifically, we explored individual differences to identify items that alter the temperature-social thought relationship. Such an exploratory approach is underused by experimental psychologists, but was very common in the early personality literature (Burish, 1984), and now again advocated by psychologists (e.g., Wagenmakers et al., 2012; Yarkoni \& Westfall, 2015). Our article is thus powerful for two reasons: First, it shows the robustness of temperature manipulations, and second, we make considerable theoretical advances by identifying a relevant individual difference moderator, a construct we call Relationship Closeness Regulation. To facilitate replication and theory generation, we placed data, materials, and a Statement of Limits of Generality online (SIPS, 2016; https://osf.io/anc5m/).

\section{Methods}


We ran three studies, in which we manipulated temperature and assessed the effect on the recollection of close others. Two of these studies were not registered before data collection and submitted to this journal. After the first round of review, we pre-registered our study and collected data only after pre-registration on the Open Science Framework. We measured people's self-reported experiences with past relationships through the Experiences in Close Relationships (ECR) as potential moderator. Because the ECR did not moderate thermoregulation effects in the past (Schilder et al., 2015), we used our first study to explore whether a specific subset of items of the ECR could be candidate for a revised scale that we aimed to create. The second study was a first confirmatory study, in which we tested our interaction in a confirmatory fashion. As methods and procedure of both studies were very comparable, we chose to report the methods and procedure together. However, since we present exploratory results in our Pilot Study and confirmatory results in Study 1, we report our results separately. Then, finally, we report our pre-registered Study 2.

\section{Participants}

For the Pilot Study, we were uncertain about the a priori effect size, and based ourselves on comparable other studies (IJzerman \& Semin, 2009). Based on an a priori power analysis with effect size Cohen's $f=.25$, power $=.8$, and independent sample t-test for two independent groups, we projected needing 128 participants. To account for potential dropout, in our Pilot Study, 141 participants $\left(M_{\mathrm{age}}=23.43\right.$ years, $S D_{\mathrm{age}}=9.03 ; 58.2 \%$ male $)$ thus (voluntarily) took part in our study. We excluded the input of those who correctly indicated the purpose of the study $(N=12)$ or had an ethnicity other than native Dutch $(N=8)$. For our Pilot Study, we used the results of 121 participants $\left(M_{\mathrm{age}}=23.66, S D_{\mathrm{age}}=9.58 ; 61.2 \%\right.$ male $)$ as input for further analysis. On the basis of the effect size of the main effect $(f=-.18)$ in the Pilot Study, we updated our power analyses, with a projected 301 participants. In Study 1, 307 
participants $(46.6 \%$ male) voluntarily took part. We excluded the input of eighty-two participants who either correctly indicated the purpose of the study $(N=29)$ or had an ethnicity other than native Dutch ( $N=53$; see IJzerman \& Semin, 2009). Our final dataset consisted of 225 participants $(49.3 \%$ male $)$.

\section{Procedure and Materials}

Participants could take part if they had 10 minutes to spare and spoke Dutch. The study was performed during a two-week time frame for Study 1 and four-week time frame for Study 2 at the Tilburg University cafeterias in the "Prisma Building" and the general student cafeteria. A total of two people at a time could participate in Study 1 and a total of four people at a time in Study 2. Participants were recruited between 09:30 am and 04:00 pm for Study 1, and for Study 2 between 10:30 am and 04:30 pm. Participants provided written informed consent before being included in the research. In both studies, participants were approached by a first experimenter and told they would participate in two separate studies: One on consumer behavior and one on psychology. When a participant decided to take part, he or she was asked to fill in the Qualtrics online questionnaire via laptop or tablet. Participants first answered the Experience in Close Relationships Questionnaire (ECR; Fraley et al., 2000). The ECR (e.g., "I'm afraid that I will lose my partner's love”) consists of 36 items and was answered on a 7-point Likert Scale ranging from 1 (Completely disagree) to 7 (Completely agree). The entire dataset is available on the project page of our OSF page (https://osf.io/anc5m/).

To reduce potential demand effects (Klein et al., 2014; Schilder et al., 2014), the manipulation of temperature was handled by a second experimenter who did not interact with the participants. Participants were then randomly assigned to the cold/warm condition. Depending on the condition, either warm or cold water was poured by the second 
experimenter into a cup from an unmarked flask out of the participant's and the other experimenter's sight. The cup was set next to the participant with the notion it would later on be used for the experiment. Via our questionnaire in Qualtrics, participants were then asked to hold a cup, filled with either warm or cold water, for 30 seconds. They were instructed that the cup was filled with water for a more realistic feel of the cup. After the participants held the cup for 30 seconds, they were asked to answer 5 questions about the appearance and feel of the cup (e.g. "I think this cup is pretty”; "I prefer this paper cup over a plastic cup.”), to keep the earlier mentioned purpose of the study (consumer behavior) realistic. The questions were answered on a 7-point Likert Scale ranging from 1 (Completely disagree) to 7 (Completely agree). The last question about the cup was an open-ended question asking if the participants would change anything about the cup.

Then, the participants were asked to write down the first five people they thought of ("Write down the first five people that come to mind right now") followed by an Inclusion of Other in Self rating how close they felt to the people they mentioned (IOS; Aron et al, 1992; IJzerman \& Semin, 2009). The IOS uses overlapping circles to represent how close one feels to another person. Participants had to select the picture that best represented the relationship with each person mentioned and we averaged the five values per person. The last part of the survey consisted of filling in demographic information and a funneled debriefing, via which we tried to discover whether participants knew the true purpose of the study. All participants were afterwards informed about the purpose of the study.

\section{Results}

\section{Unregistered Pilot Study}

Confirmatory Results. We ran an independent samples t-test examining and found that participants in the warm condition recalled persons marginally significantly closer to 
themselves $(M=4.45, S D=1.18)$ compared to participants in the cold condition $(M=4.06$, $S D=.99), d=0.39($ CI $95[-.02, .72]), t(119)=-1.97, p=.051$.

Exploratory Results. We were interested in whether ECR moderated the relationship between recall of close others. We did so in two phases. In the first phase, we used the traditional ECR scale, which includes the entire range of attachment items (ECR; Study $1 \alpha=$ .91 , Study $2 \alpha=.94$ ) and two subscales (anxiety; Study $1 \alpha=.90$, Study $2 \alpha=.91$; avoidance Study $1 \alpha=.86$, Study $2 \alpha=.91)$. In the second phase, we planned to explore items and their potential differences across conditions. In earlier research (Schilder et al., 2014) we had not detected differences using the entire ECR or the pre-set subscales, thus we already had this initial strategy to explore a subset of items in Study 1, which we could then confirm in Study 2.

First Phase. Via a stepwise multiple regression analysis, with the average of the standardized items of the ECR and temperature condition (coded -1 and 1) we found no significant interaction $(\beta=-.001, t(120)=-.04, p=.97, s r=-0.003)$. We also found no significant interaction between temperature condition and the anxiety subscale $(\beta=-.007$, $t(120)=-0.52, p=.61, s r=-.05$,$) or the avoidance subscale (\beta=-.005, t(120)=-0.40, p=.69$, $s r=-.04)$.

Second Phase. Previous work (Schilder et al., 2015) and these analyses here did not reveal moderation via the full ECR and its known subscales. Other, previous work that relied on different scales did show this moderation (see e.g., Fay \& Maner, 2012; IJzerman, Karremans, Thomsen, \& Schubert, 2013). Thus, we set forth to explore whether the lack of moderation was due to insensitivity of the ECR to pick up on these effects, and whether a shorter version could pick up on the effects (see e.g., Wei, Russell, Mallinckrodt, \& Vogel, 2007). 
In this inductive approach, we conducted 36 separate regression analyses for each specific item of the scale with standardized score of each item, condition, and their interaction terms. We then selected 15 items with interaction $B>.10$, and plotted each interaction. We searched for a moderator based on the data and plots alone. This data driven approach showed us four interaction patterns, which we then turned into four different subscales.

We thus now tested the interactions for each scale. This gave a significant interaction effect for the subscale $(B=.43, t(117)=2.90, p<.01, s r=.26)$, consisting of the following three items: "My romantic partner makes me doubt myself”; "I feel comfortable sharing my private thoughts and feelings with my partner"; "I find it easy to depend on romantic partners" $(\alpha=.44)$. From here on, we refer to this new subscale as 'Relationship Closeness Regulation' (RCR). Note that the scoring is somewhat counterintuitive; in the original ECR higher scores denote greater insecurity. Thus, lower scores on the RCR denote greater closeness. We found only marginally/ns significant effects for our second $(\alpha=.36 ; B=-.22, t 117)=-1.73, p=.09$, $s r=-.16)$, third $(\alpha=.70 ; B=.28, t(117)=1.93, p=.06, s r=.18)$, and fourth subscale $(\alpha=$ $.63 ; B=-.30, t(117)=-1.96, p=.05, s r=-.18){ }^{1}$

\section{Confirmatory Results Unregistered Study 1}

To test the robustness of the RCR scale as a moderator in the relationship between temperature and the recollection of close others, we ran a second, confirmatory study. We again found an interaction between temperature condition and the Relationship Closeness Regulation scale in predicting the accessibility of close others $(\alpha=.36 ; B=.38, t(219)=3.12$, $p<.01, s r=.21)$. In line with the (marginally/ns significant) effects of Pilot Study, we found no effect for the second subscale $(\alpha=.57 ; B=.15, t(219)=1.58, p=.12, s r=.11)$, the third subscale $(\alpha=.71 ; B=.09, t(219)=.72, p=.47, s r=.05)$, and the fourth subscale $(\alpha=.57 ; B$

\footnotetext{
${ }^{1}$ Note that the use of the p-value is not entirely correct when doing exploratory research and should thus not be taken at face value for meta-analyses. The effect sizes and p-values helped inform us which variables to rely on for further confirmatory testing.
} 


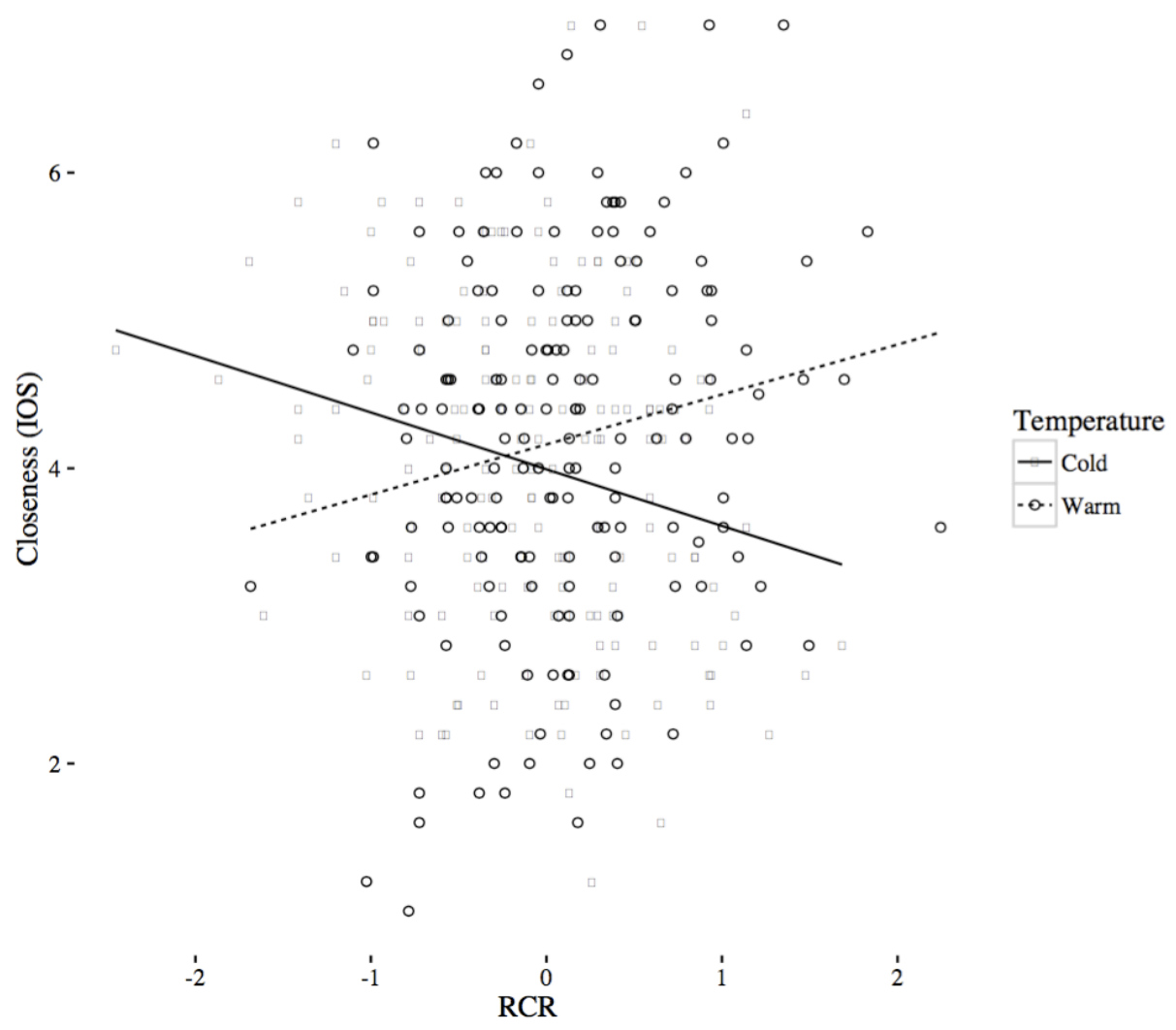

Figure 1. Interaction between Relationship Closeness Regulation and temperature condition in a Dutch sample, created in ggplot2 (Wickham \& Chang, 2016). Higher scores on the RCR indicate less closeness in the relationship regulation orientation.

$=.03, t(219)=.23, p=.82, s r=.02)$. Our results in Study 2 thus confirmed our results of Study 1.

\section{"Meso"-Analysis}

To interpret the interaction effect and to generate an effect size for the power calculation for our follow-up study, we then ran a "meso-analysis" of the two Dutch samples with the same regression models, including "StudyID" as predictor. Importantly, there was no significant threeway interaction between RCR, StudyID, and condition $(B=-.046, t(336)=$ $.23, p=.82, s r=-.01$ ), which is why we could interpret the significant twoway interaction between RCR and condition $(B=.40, t(340)=4.20, p<.01, s r=.22)$, see Figure 1 . When 
centering RCR at 1SD above the mean (=low in relationship closeness orientation), participants in the warm condition thought of closer others than participants in the cold condition, $B=.44, t(340)=3.94, p<.01, s r=.21$. This was exactly the opposite for those 1SD below the mean (=high in relationship closeness orientation), participants in the warm condition thought of more distant others than participants in the cold condition, $B=-.28$, $t(340)=-2.40, p=.02, s r=-.13$.

For our second subscale there was a significant threeway interaction with StudyID ( $B$ $=.37, \mathrm{t}(336)=2.25, p=.03, s r=.12)$, rendering the effect between the second subscale and condition uninterpretable. There were no significant threeway interaction effects for the third $(B=-.20, t(336)=-.99, p=.33, s r=-.05)$ and fourth subscales $(B=.33, t(336)=1.60, p=$ $.11, s r=.09)$, but also no significant twoway interactions between the third $(B=.16, t(340)=$ $1.64, p=.10, s r .09)$ and fourth subscale $(B=-.09, t(340)=-.90, p=.37, s r=-.05)$ and condition. The picture that we got from our exploratory and confirmatory analyses thus remained intact.

\section{Preregistered Study 2}

After first review with this journal, we were asked to run a pre-registered replication in an independent sample. We ran a replication, which was pre-registered at the Open Science Framework (https://osf.io/h82um/). The study was run in Grenoble, France, as the first author had moved universities in the meantime.

\section{Methods}

\section{Procedure and Materials}

The procedure was identical to the first two studies, with two exceptions: We added a different order of the manipulation and measurement of dependent variable, where we asked to think about people prior to the temperature manipulation. By first asking to think about 
people, we could test the alternative hypothesis whether people would feel closer to those people they had mentioned prior to the temperature manipulation.

Because the RCR suffered from a poor reliability in our Pilot Study and Study 1, after Study 1 we had started developing the 29-item Social Thermoregulation and Risk Avoidance Questionnaire (STRAQ-1; Vergara et al., 2018). We developed the scale with the presupposition that people bond with others for (at least) two reasons: To regulate temperature and to reduce (social) risks. The measure taps into the desire to outsource temperature regulation (e.g., "When I feel cold I seek someone to cuddle with") and to avoid social risk (“I try to maintain myself in familiar places"). In the 12-country sample in which we validated and tested the measure, especially the social thermoregulation subscale was reliable (average $\alpha=.77)$ and related reliably to attachment avoidance $(r=-.32)$. We asked the STRAQ- 1 one week after the experiment. The remainder of the experiment remained identical. The reliability of the RCR was comparable $(\alpha=.41)$.

\section{Participants: Registered}

We conducted an a priori power analysis, based on the partial correlation of our interaction from our "meso"-analysis of .22 (corresponding to an effect size of Cohen's $f=$ .226). Based on an alpha of .05, power of .95 , and 2 tested predictors in a multiple regression, we thought we would need 72 participants. In order to test the "order" effect, and based on an alpha of .05 , power of .95 , we thought we would need 257 participants (based on an analysis of variance with 8 groups). Because we had a drop-out of $26.7 \%$ in our prior studies, we tried to overshoot our sample and reach 350 participants. Note however that we later discovered that we were mistaken with our sample size calculation for the interaction without order (see Giner-Sorolla, 2018) and that an experiment with 72 participants would have been underpowered. 


\section{Changes from the Pre-Registration to the Actual Study}

After a first review at this journal, we pre-registered our study on the Open Science Framework (link to pre-registration: https://osf.io/h82um/ and to pre-registration form: https://osf.io/h82um/register/565fb3678c5e4a66b5582f67\#q23). Compared to the preregistration, we changed some (relatively minor) aspects:

- Because the first author moved from the Netherlands to France, participants were French (and not Dutch).

- Asking about ethnicity is not allowed in France. Although the first author mistakenly asked for ethnicity as he was unaware of this legislation, we deleted the data about ethnicity without using it. We used nationality as a proxy for cultural group instead.

- We changed our analysis script, because we re-programmed the questionnaire (after the first author's move to France).

\section{Participants: Actual Sample}

We excluded all participants with a nationality other than French $(N=16)$. None of the participants correctly guessed the purpose of the study. For Study 3, we used the data of 350 participants $\left(M_{\text {age }}=20.32, S D_{\text {age }}=2.92 ; 80.9 \%\right.$ female $)$ who voluntarily took part in our study. We collected 17 participants more than planned as we could not pull our study from the online registration for our last data collection day. Furthermore, because we sent the STRAQ a week after, we a drop-out for the second questionnaire of $35.9 \%$. The total number of participants for the sample including the STRAQ was 225. We recorded water temperature from time-to-time, and posted the measurement on our project page (https://osf.io/8z6j7/). 


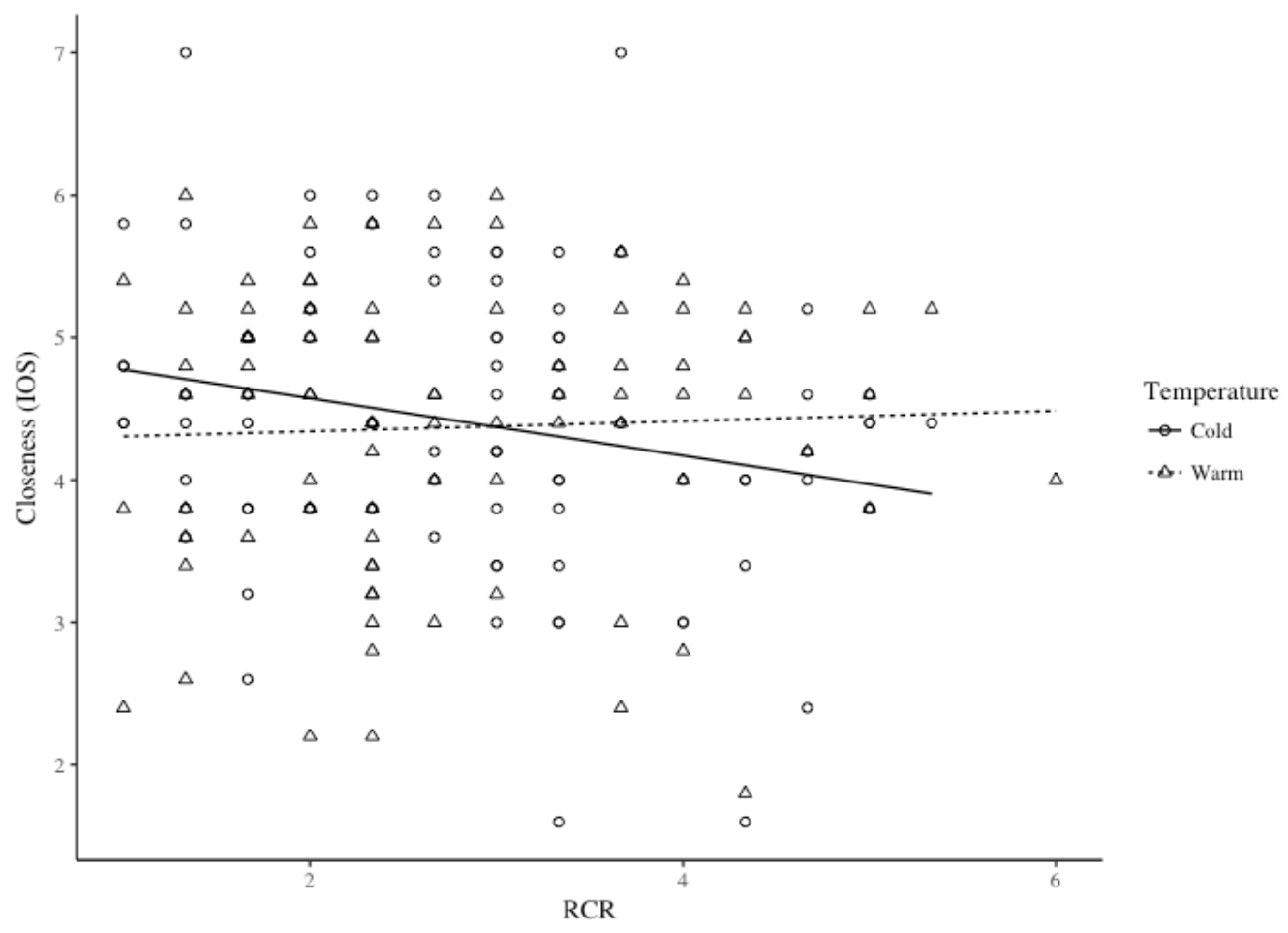

Figure 2. Interaction between Relationship Closeness Regulation and temperature condition in a French sample, created in ggplot2 (Wickham \& Chang, 2016). Higher scores on the RCR indicate less closeness in the relationship regulation orientation.

\section{Confirmatory Results Preregistered Study 2}

\section{Replication Results: Manipulation before recall}

In our pre-registered replication study in a French sample, we again found an interaction between temperature condition and the Relationship Closeness Regulation scale in predicting the accessibility of close others $(B=0.24, t(168)=1.80, p=.04)$, that the lower the $\mathrm{RCR}$ (= more past positive relationship experiences) related to more closeness to the people that participants were currently thinking off $(B=-0.44, t(168)=-2.14, p=.02)$, and colder (versus warmer) temperatures let participants think more of people who are close to them ( $B$ $=-0.71, t(168)=-1.78, p=.04){ }^{2,3}$ The interaction was comparable (though weaker) as

'One reviewer had asked us when we would expect a main effect. In reply, we pre-registered a main effect of temperature condition if the RCR was equally distributed across temperature conditions (by that we meant that means would not significantly different; see also https://osf.io/h82um/register/565fb3678c5e4a66b5582f67\#q23). We first tested whether - in our confirmatory 
compared to the Pilot Study and Study 2 (see also Figure 2). When we tested the simple slopes, we observed a difference for those scoring low versus high on the RCR for the cold condition (Est. $=-0.20, \mathrm{SE}=0.09, p=.02)$ and not in the warm condition $($ Est. $=0.04, \mathrm{SE}=$ $0.09, p=.36)$. We observed no differences on who people were thinking of for individuals low $($ Est. $=-.32, \mathrm{SE}=0.21, p=.07)$, medium $($ Est. $=-0.04, \mathrm{SE}=0.15, p=.39)$ or high $($ Est. $=$ $0.23, \mathrm{SE}=0.21, p=.14)$ on the RCR.

\section{Additional Confirmatory Analyses on Order}

We did not have a strong prior regarding the second order (recall before manipulation), but we suspected the manipulation would not return the same effect. We suspected that changing the nature of an existing relationship requires more than simply holding a cold/warm cup. Our suspicion was confirmed: There was no main effect of temperature $(B=0.06, t(174)=.13, p=.90)$, nor an interaction effect between the RCR and temperature $(B=-0.07, t(174)=-.04, p=.66)$. We also ran the threeway interaction with temperature condition, the RCR, and order included. This was not significant $(B=-0.30$, $t(342)=-1.51, p=.07)$. When we repeated the replication interaction analyses (but now with the entire sample), the effect decreased somewhat in strength and became marginally/nonsignificant $(B=0.24, t(342)=1.64, p=.05)$. We suspect that the threeway interaction was not significant because the study was too underpowered to detect a threeway interaction, while the interaction became non-significant because we introduced noise with the second (less

\footnotetext{
dataset - the RCR means were different for the temperature conditions. They were not $(B=-0.11, t(170)=-0.60$, $p=.55$ ). Thus, we expected a main effect of temperature, which we found.

:Because we replicated the original effect, we pre-registered that we would do a one-tailed test (see https://osf.io/h82um/register/565fb3678c5e4a66b5582f67\#q23). All analyses here reported in our confirmatory section are thus one-tailed.
} 


\section{Influential Obs by Cooks distance}

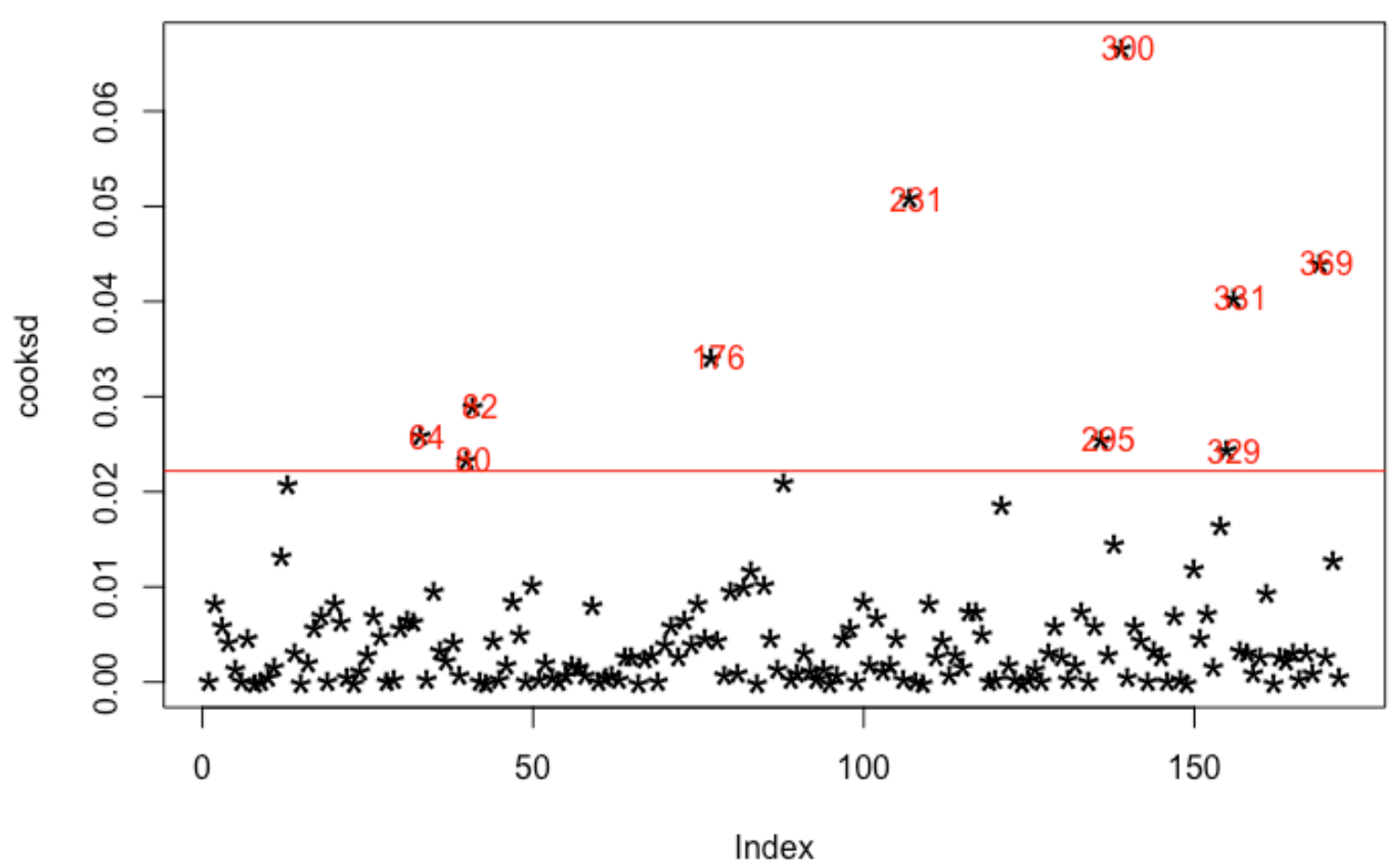

Figure 3. Influential observations detected through Cook's distance. The model was our replication interaction model.

sensible) manipulation.

\section{Exploratory Analyses ${ }^{4}$}

As we had pre-registered our tests, we had committed to an analysis plan a priori.

Nevertheless, the $p$-value that we had obtained could be indicative of an effect that is not true. In order to ascertain that our effects were not due to a few influential cases, we also conducted an exploratory analysis using Cook's distance (excluding outliers with $4 *$ Cook Distance mean), based on our interaction model for the replication. Our outlier analysis suggested we should exclude 10 more participants (see also Figure 3). When we repeated our interaction

\footnotetext{
"Exploratory analyses were run only with the "replication order" (where the manipulation preceded recall), with the exception of the reliability analyses and the correlations between the Social Thermoregulation subscale of the STRAQ and the attachment subscales).
} 
analyses without those 10 cases, our interaction effect was nearly identical $B=0.23, t(165)=$ $1.72, p=.04) .{ }^{5}$ We also included temperature of the day to get a better understanding of outside temperature in this relationship. The interaction effect was also nearly identical when we controlled for temperature in Grenoble in the period of day $(B=0.25, t(162)=1.87, p=$ $.03)$, the minimum temperature of the day $(B=0.24, t(162)=1.82, p=04)$, and the maximum temperature of the day $(B=0.24, t(162)=1.82, p=.04)$ when the participant took part.

It is clear that the RCR subscale suffers from low reliabilities throughout our pilot study and our two replication studies. After our Study 1, we therefore started to develop the STRAQ-1, which assesses social thermoregulation-based attachment desires. We ran exploratory analyses using this Social Thermoregulation subscale of the STRAQ-1. Like in its original, the subscale was sufficiently reliable $(\alpha=.74$, Omega hierarchical $=.54)$. The Social Thermoregulation subscale correlated negatively with the $\operatorname{RCR}(r=-.23, p<.001, N=225)$, with the avoidance $(r=-.30, p<.001, N=225)$ and positively with the anxiety $(r=.15, p=$ $.03, N=225$ ) subscales of the ECR, further confirming that social thermoregulation relates to attachment motives. Although the effect size was bigger than for Study 2's RCR moderation, when we ran the interaction between Social Thermoregulation and temperature, we detected no significant effects $(B=0.37, t(107)=1.52, p=.13$ (two-tailed). We suspect the nonsignificant effect for the interaction with the Social Thermoregulation subscale to be mostly due to this analysis to be underpowered due to the high drop-out from session 1 to 2 .

\section{Discussion}

Results of an initial two studies showed strong support for our overall expectation: Temperature affects the cognitive accessibiility of loved ones, and the nature of these

\footnotetext{
Even though no participants truly guessed the purpose of the experiment, there were 15 participants who guessed the opposite direction (warmth leads to thinking about people who are closer) and 16 participants who did not specify a direction (the temperature condition leads to thinking about different people). When we reran the analyses with the former (one-tailed) we found the same interaction $(B=0.30, t(156)=2.21, p=.01)$ and the same was true for the latter $(B=0.30, t(154)=2.15, p=.02)$.
} 
thoughts depends on people's prior expectation in past relationships. Specifically, we found that people who have positive experiences in past relationships are more likely to think of close others after holding something cold (vs. warm), while the opposite is true for those who have negative past relationship experiences. At the request of this journal, we then preregistered and replicated the study. We replicated the interaction effect of the first two studies in our Study 3.

These findings are in line with past literature showing that people with poorer interpersonal skills may respond more strongly to warmth as a social cue. Specifically, Fay and Maner (2012) found that "warm" (vs. cold) anxiously attached people are especially likely to perceive objects as closer. However, our findings are not in line with priming literature in social thermoregulation (e.g., greater generosity of securely attached children in warm vs. cold conditions; IJzerman et al., 2013), and we had therefore not predicted them as such. An alternative way of interpreting the current findings is by understanding these as compensatory effects. Specifically, after holding a cold cup, people with positive past experiences in relationships are more likely to remember of close others to answer their need to affiliate, an effect that we have found consistently in (pre-registered) studies the colder the environment (Van Acker et al., 2016). Note that the effects of coldness were consistent across our three studies (and this was not true for warmth). Post-hoc, one can make sense of these findings, as animals (including humans) primarily outsource temperature regulation when cold. It could be that "priming" effects of temperature are restricted to specific situations, for people who have formed an internal model that warmth means affection (i.e., the securely attached).

There are caveats. We recognize our exploratory approach to be simple; yet, our confirmatory tests in Study 2 and in our pre-registered Study 3 demonstrated it was effective. 
Before we pre-registered our Study 3, there was the (unlikely) hypothesis that temperature affects how close others are perceived (rather than that close others become more accessible in colder conditions). We could not find confirmation for this idea when we first asked people about others and then manipulated temperature. As the idea was not very plausible, and we found no evidence for this alternative hypothesis, we conclude that thinking about loved ones is a self-regulatory mechanism against the cold.

Furthermore, the effect size decreased from the Pilot Study $(B=.43) 1$ and $2(B=.38$; summary $B=.40$ ) to Study 3 (the $B$ value was around .24 , depending on the analysis). One explanation is that we forced the moderation in the Pilot Study and exaggerated the effect size. This could be true if one overfits and finds an effect size in a small sample (with no replication). This was not true for us: Our sample size was considerable and we replicated the effect. Chances of overfitting are thus small and even for the pilot study, we likely detected the true effect size.

We think instead that the distance from the equator is a reasonable explanation for the smaller effect size. First of all, in the Human Penguin Project, we found that people further away from the equator have a lower core body temperature and that their social network buffers their core body temperature from lower temperatures (IJzerman et al., 2018). Second, we found our meta-analysis that social thermoregulation effect sizes are moderated by latitude, such that studies conducted further from the equator have larger effect sizes (IJzerman, Hadi, Neyroud, Klein, \& Ropovik, 2018). This replication is consistent with that meta-analysis: The effect sizes $(B=0.40)$ in Tilburg (the Netherlands; latitude of 51.56 degrees North of the equator) are smaller than the one $(B=0.24)$ in Grenoble (France; latitude of 45.18 degrees North of the equator). Distance from the equator appears to be a reasonable explanation for why the effect size dropped from Studies 1 and 2 to 3 . This likely also 
explains why the simple slopes were not significant. Note that we had included this explanation in our Limits of Generality prior to running our final study (https://osf.io/anc5m/). At that time however, we had no information to predict the differences in effect size. The current study will help us do so in future research.

Finally, even though we find significant moderation, the reliability of the RCR was mediocre. From the outset, we had not expected the ECR to moderate social thermoregulation effects as we did not find moderation using the ECR in past work (e.g., Schilder et al., 2014). We suspect that the ECR is not appropriate in measuring biological motives and desires (like social thermoregulation) underlying attachment needs. From a scientific perspective, using the ECR is most appropriate when trying to extract patterns of behavior and cognition. We did so and still picked up a replicable pattern. However, we reason that this is not because of the strengths of the ECR/RCR, but despite the problems with the link between the ECR and social thermoregulation and the mediocre reliability of the RCR. That means that better measures need to be developed if one wants to formalize predictions related to social thermoregulation (as with our latitude-effect size prediction). Although the ECR has been very useful in many domains, to study the origins of one's attachment motives, should one perhaps not instead focus on assessing biological drives underlying attachment (like wanting to cuddle with someone when feeling cold)? We did a first exploratory analysis to. Our Social Thermoregulation was more reliable $(\alpha=.74$ as compared to RCR $\alpha=.41)$ and correlated with the RCR (as well as attachment avoidance). Although non-significant (due to high drop out), the measure shows promise. Further, the relation between the RCR and the Social Thermoregulation further attests to the robustness of our findings.

We found reliable support for the idea that a temperature manipulation makes close others more accessible, at least for those who worry more about their relationships. Though 
doubts in the literature have arisen, a considerable amount of high-powered reports exists relating temperature to our social life. Should warmth-based therapies be further explored to make relationship therapy more efficacious (IJzerman et al., 2017)? Our answer is a resounding yes. But exactly how this should be implemented requires much further research. 


\section{Author Note}

The preparation of this work was partly funded by a NWO Veni grant (016.145.049) and a French National Research Agency “Investissements d'avenir” program grant (ANR-15IDEX-02) both awarded to Hans IJzerman. Hans IJzerman (HIJ) developed the study concept. Testing and data collection were performed under HIJ's supervision. HIJ and Michel Schrama (MS) performed analyses and interpretation of the first two studies. HIJ performed analyses and interpretation of the final study. HIJ and MS drafted the first manuscript, and Tila Pronk (TP) and Jorick Post (JP) provided multiple critical revisions. Jorick Post (JP), Lison Neyroud (LN), and Rémi Courset (RC) were involved in the preparation of our pre-registered study and LN and RC collected the data for this study. LN and RC provided critical revisions for the final paper and RC did code review for the final study. The project page for the first two studies is available at the Open Science Framework: https://osf.io/anc5m/. We forked this project for our pre-registration: https://osf.io/bwh26/ (analysis code: https://osf.io/2rv7b/). We thank Dominique Muller, Brent Roberts, the editor Anthony Freitas, and two anonymous reviewers for their helpful comments on our article. All remaining errors are the authors' responsibility. 


\section{Appendix A: Social Thermoregulation Items}

1. I usually have more physical contact with others than most people

2. When people are close to me, I like to be really close to them

3. When I feel cold I seek someone to cuddle with

4. I like to warm up my hands or feet by touching someone who I am close to

5. I prefer to warm up with someone rather than with something

6. I love to share a hot beverage with a friend when I am feeling down

7. When I am distressed I really need a "warm shoulder"

Appendix Note: The STRAQ-1 is currently still under development. Please contact the first author for the latest STRAQ-1 version. 


\section{References}

Aron, A., Aron, E. N., \& Smollan, D. (1992). Inclusion of Other in the Self Scale and the structure of interpersonal closeness. Journal of Personality and Social Psychology, 63, 596.

Burish, M. (1984). Approaches to personality inventory construction. A comparison of merits. American Psychologist, 39, 214 - 227.

Dotsch, R., \& Todorov, A. (2012). Reverse correlating social face perception. Social Psychology and Personality Science, 3, 562-571.

Ebensperger, L. A. (2001). A review of the evolutionary causes of rodent group-living. Acta Theriologica, 46, 115-144.

Fay, A. J., \& Maner, J. K. (2012). Warmth, spatial proximity, and social attachment: The embodied perception of a social metaphor. Journal of Experimental Social Psychology, 48, 1369-1372.

Fraley, R. C., Waller, N. G., \& Brennan, K. A. (2000). An item-response theory analysis of self-report measures of adult attachment. Journal of Personality and Social Psychology, 78, 350-365.

IJzerman, H., \& Semin, G. R. (2009). The thermometer of social relations: Mapping social proximity on temperature. Psychological Science, 10, 1214-1220.

IJzerman, H., Janssen, J. A., \& Coan, J. A. (2014). Maintaining warm, trusting relationships with brands: Increased temperature perceptions after thinking of communal brands. Plos One. 
IJzerman, H., Heine, E. C., Nagel, S. K., \& Pronk, T. M. (2017). Modernizing relationship therapy through Social Thermoregulation Theory: Evidence, hypotheses, and explorations. Frontiers in Psychology, 8, 635.

IJzerman, H., Karremans, J., Thomsen, L., \& Schubert, T. W. (2013). Caring for sharing: How attachment styles modulate communal cues of physical warmth. Social Psychology, 44, 160-166.

IJzerman, H., Hadi, R., Neyroud, L., Klein, R. A., \& Ropovik, I. (2018). Social thermoregulation: A meta-analysis. Manuscript in preparation.

IJzerman, H., Coan, J. A., Wagemans, F. A. M., Missler, M. A., Van Beest, I., Lindenberg, S. M., \& Tops, M. (2015). A theory of social thermoregulation in human primates. Frontiers in Psychology.

IJzerman, H., Lindenberg, S., Dalgar, I., Weissgerber, S. C., Vergara, R. C., \& Zickfeld, J. (2018). The Human Penguin Project: Climate, social integration, and core body temperature. Preprint at https://doi. org/10.17605/OSF. IO/6B7NE.

Inagaki, T., Irwin, M. R., Moieni, M., Jevtic, I., \& Eisenberger, N., I. (in press). A pilot study examining physical and social warmth: Higher (normal) body temperature is associated with greater feelings of social connection. Plos One.

Inagaki, T. K., Irwin, M. R., \& Eisenberger, N. I. (2015). Blocking opioids attenuates physical warmth-induced feelings of social connection. Emotion.

Janssen, C. W., Lowry, C. A., Mehl, M. R., Allen, J. J., Kelly, K. L., Gartner, D. E., ... \& Fridman, A. (2016). Whole-body hyperthermia for the treatment of Major Depressive Disorder: A Randomized Clinical Trial. JAMA Psychiatry.

Klein, O., Doyen, S., Leys, C., Magalhäes de Saldanha da Gama, P. A., Miller, S., Questienne, L., \& Cleeremans, A. (2012). Low hopes, high expectations: Expectancy 
effects and the replicability of behavioral experiments. Perspectives on Psychological Science, 7, 572 - 584.

LeBel, E. P., \& Campbell, L. (2013). Heightened sensitivity to temperature cues in individuals with high anxious attachment: Real or elusive phenomenon? Psychological Science, 24, 2128-2130.

Lynott, D., Corker, K. S., Wortman, J., Connell, L., Donnellan, B. M., Lucas, R. E., \& O'brien, K. (2014). Replication of “experiencing Physical Warmth Promotes Interpersonal Warmth" bywilliams and Bargh (2008). Social Psychology, 45, 216-222.

McFarland, R., Fuller, A., Hetem, R. S., Mitchell, D., Maloney, S. K., Henzi, S. P., \& Barrett, L. (2015). Social integration confers thermal benefits in a gregarious primate. Journal of Animal Ecology, 84, 871- 878.

Simonsohn, U., Nelson, L. D., \& Simmons, J. P. (2014). P-curve: A key to the file drawer. Journal of Experimental Psychology: General, 143, 534 - 547.

Schilder, J. D., IJzerman, H., \& Denissen, J. J. A. (2014). Physical warmth and perceptual focus: A replication of IJzerman and Semin (2009). Plos One.

SIPS (2016). SLOG White Paper. Retrieved from http://bit.ly/290ngb0.

Van Acker, B. B., Kerselaers, K., Pantophlet, J., \& IJzerman, H. (in press). Homelike thermoregulation: How physical coldness makes an advertised house into a home. Journal of Experimental Social Psychology.

Vess, M. (2012). Warm thoughts: Attachment anxiety and sensitivity to temperature cues. Psychological Science, 23, 472-474.

Vergara, R. C., Hernández, C., Jaume-Guazzini, F., Lindenberg, S., Klein, R. A., \& IJzerman, H. (2018). Development and validation of the Social Thermoregulation and Risk Avoidance Questionnaire (STRAQ-1). Manuscript in preparation. 
Wagenmakers, E.-J., Wetzels, R., Borsboom, D., Maas, H. L. J. van der, \& Kievit, R. A. (2012). An Agenda for Purely Confirmatory Research. Perspectives on Psychological Science, 7, 632-638.

Wickham, H., \& Chang, W. (2016). Package ggplot2. Retrieved from https://cran.rproject.org/web/packages/ggplot2/ggplot2.pdf.

Williams L. E., \& Bargh J. A. (2008). Experiencing physical warmth promotes interpersonal warmth. Science, 322, 606-607.

Zhang, Y., \& Risen, J. L. (2014). Embodied motivation: Using a goal systems framework to understand the preference for social and physical warmth. Journal of Personality and Social Psychology, 107, $965-977$ 